\title{
Online Monitoring of Superoxide Anions Released from Skeletal Muscle Cells Using an Electrochemical Biosensor Based on Thick-Film Nanoporous Gold
}

\author{
Ramin Banan Sadeghian, ${ }^{\dagger}, \dagger \dagger$ Serge Ostrovidov, ${ }^{\dagger}$ Jiuhui Han, ${ }^{\dagger}$ Sahar Salehi, ${ }^{\dagger}$ \\ Behzad Bahraminejad,,$\neq \dagger \dagger$ Hojae Bae, ${ }^{\S}$ Mingwei Chen ${ }^{\dagger}{ }^{\dagger}$ Ali Khademhosseini ${ }^{*}, \dagger, \S, \uparrow,+\neq, \#, \uparrow$ \\ $\dagger$ WPI-Advanced Institute for Materials Research, Tohoku University, Sendai 980-8577, Japan \\ $¥$ Department of Electrical Engineering, Faculty of Engineering, Majlesi Branch, Islamic Azad Univer- \\ sity, Esfahan 86316-56451, Iran \\ $\S$ College of Animal Bioscience and Technology, Department of Bioindustrial Technologies, Konkuk \\ University, Hwayang-dong, Kwangjin-gu, Seoul 143-701, Republic of Korea \\ †† Biomaterials Innovation Research Center, Division of Biomedical Engineering, Department of Medi- \\ cine, Brigham and Women's Hospital, Harvard Medical School, Boston, Massachusetts o2139, United \\ States \\ \# Harvard-Massachusetts Institute of Technology Division of Health Sciences and Technology, Mas- \\ sachusetts Institute of Technology, Cambridge, Massachusetts 02139, United States \\ \# Wyss Institute for Biologically Inspired Engineering, Harvard University, Boston, Massachusetts \\ 02115, United States \\ ^ Department of Physics, Faculty of Science, King Abdulaziz University, Jeddah 21569, Saudi Arabia \\ KEYWORDS: electrochemical biosensor, nanoporous gold, reactive oxygen species, skeletal muscle \\ cells, superoxide anions
}


Supporting Information for Publication

\section{TABLE OF CONTENTS}

Figure S1. Schematic illustration of the NPG-covered electrode fabrication process steps.

Figure S2. A sample cyclic voltammogram recorded from an NPG-covered electrode for the purpose of computing the electrode electrochemically active surface area (ESA).

Figure $S_{3}$. Electrochemical measurement setup/scheme.

Figure $\mathrm{S}_{4}$. Evidence of cyt-c immobilization on gold electrodes.

On sensor calibration using the xanthine-XOD system.

On the dependence of electrode sensitivity upon surface coating

On evaluating the low limits of detection

Figure $\mathrm{S}_{5}$. Cross-sectional SEM images snapped from the electrodes of both kind after cyt-c immobilization.

Figure S6. EDS map of the NPG-covered electrode cross-section with cyt-c.

Figure $\mathrm{S}_{7}$. $\mathrm{C}_{2} \mathrm{C}_{12}$ cells seeded on polyester porous membranes.

Figure S8. Biosensor specificity demonstrated by the superoxide-scavenging effect of SOD. 


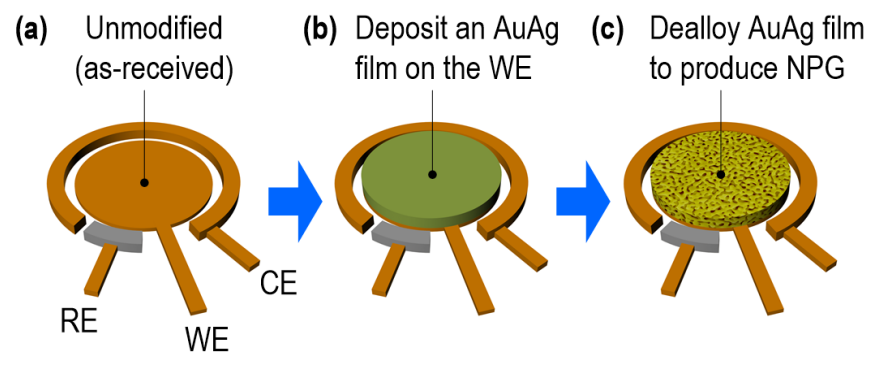

Figure S1. Schematic illustration of the NPG-covered electrode fabrication process steps. The working electrode (WE) of screenprinted strips as-received (a) are electrodeposited with a film of AuAg (b), which is subsequently rendered into NPG by chemical dealloying (c). RE and CE label the reference and the counter electrodes respectively.

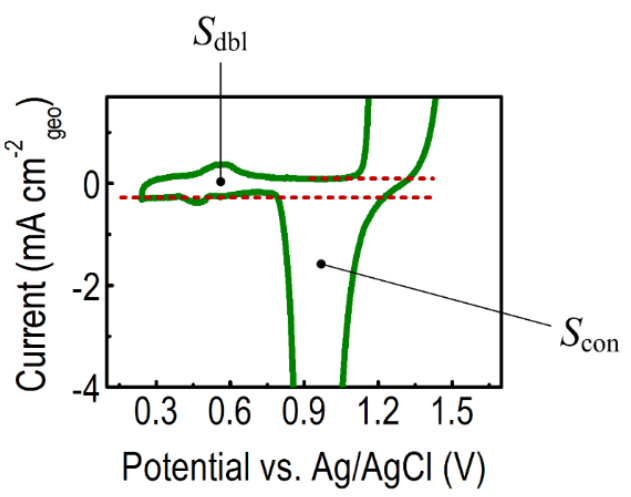

Figure S2. A sample cyclic voltammogram recorded from an NPG-covered electrode for the purpose of computing the electrode electrochemically active surface area (ESA). $S_{\mathrm{con}}$ is the area of the reduction peak and corresponds to the consumed charge. $S_{\mathrm{db}}$ is the area of the double-layer capacitance charge region of the curve. The consumed and double-layer charges are given by $C_{\text {con }}=$ $S_{\mathrm{con}} / v$ and $C_{\mathrm{dbl}}=S_{\mathrm{dbl}} / v$ in $\mu \mathrm{C}$ respectively, where $v$ is the scan rate. The ESA, expressed in $\mathrm{cm}^{2}$, is given by ESA $=\left(C_{\mathrm{con}}-C_{\mathrm{dbl}}\right) /$ $350 \mu \mathrm{C} \mathrm{cm}{ }^{-2}$.

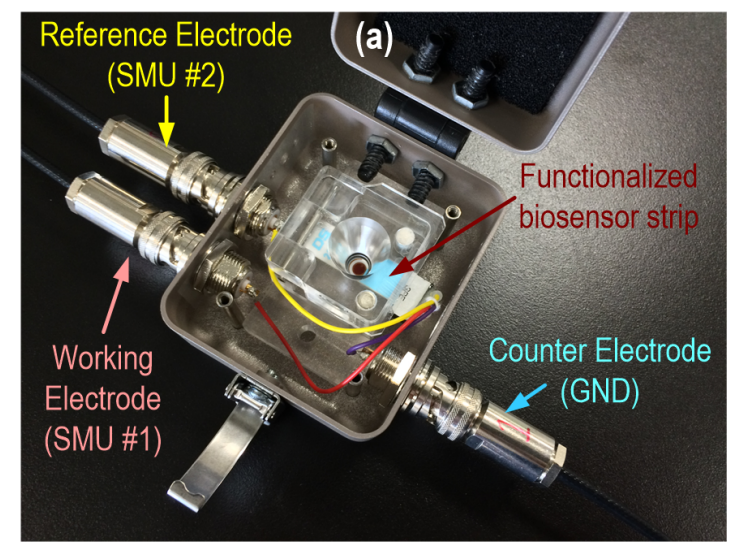

(b)

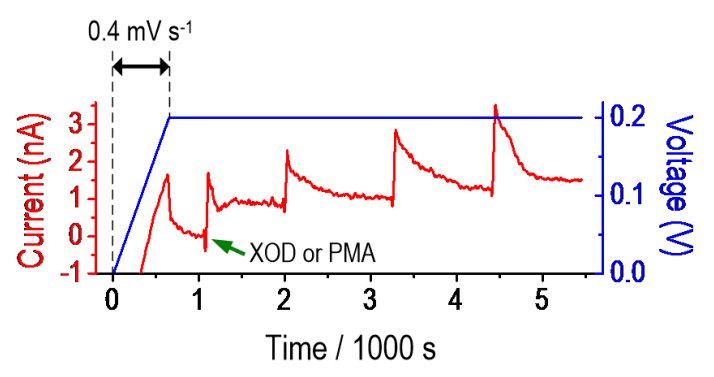

Figure S3. Electrochemical measurement setup. Porous polyester membranes were transferred onto the biosensor strips secured in a transparent methacrylate wall-jet flow-cell (DropSens, Spain). The flow-cell was placed inside a custom-made fixture with three electrical feedthroughs. It was filled with $500 \mu \mathrm{L}$ of either saturated xanthine (Sigma, USA) solution for sensor calibration (no cells) or pure HEPES (for live cells) during extracellular superoxide measurements. The working and reference electrodes were individually wired to two source-measure units of a Keithley 4200 semiconductor parameter analyzer. The counter electrode was connected to the ground unit of the tool. All connections were made through guarded triax cables. Amperograms where normally recorded at a working electrode voltage of $200 \mathrm{mV}$ versus the Ag reference electrode unless noted otherwise. (a) Biosensor fixture with electrical feedthroughs. (b) A sample calibration chronoamperogram demonstrating our low-level measurement strategy. In order to avoid capacitive current rush and reduce the settling time, the working electrode voltage was ramped at $0.4 \mathrm{mV} \mathrm{s}^{-1}$ up to the bias voltage of $200 \mathrm{mV}$. Bolus of XOD (X450o, Sigma, USA) for calibration, or PMA (Sigma, USA) to stimulate the cells was added as soon as current achieved a steady-state after about 1000 sec as indicated by an arrow. 

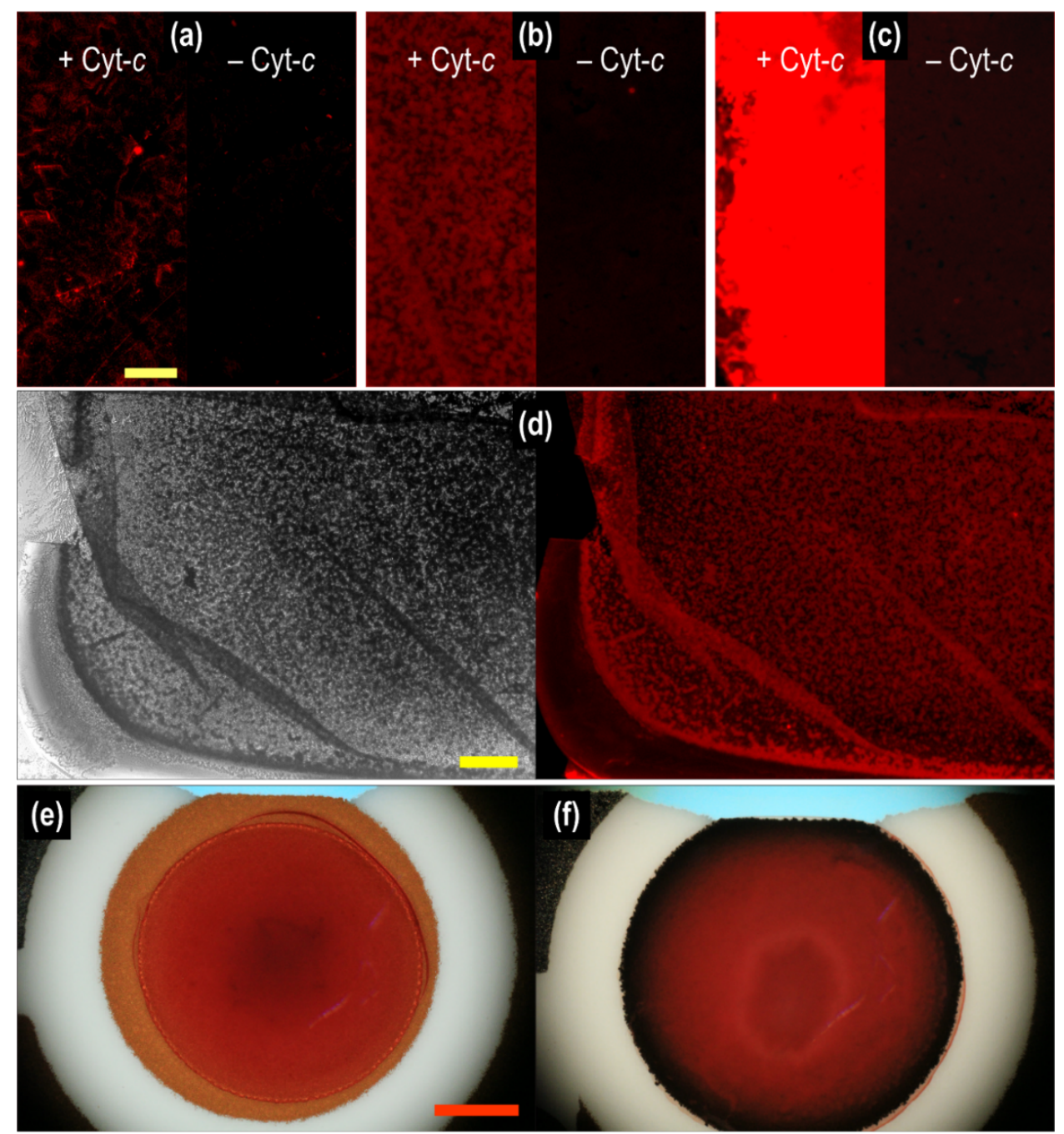

Figure S4. Evidence of cyt-c being immobilized on gold electrodes. Monoclonal mouse anti-cyt-c antibody, ab115266 (Abcam, USA), diluted 100 times in o.1\% bovine serum albumin (BSA) (Sigma, USA) was used to fingerprint cyt-c immobilized on gold electrodes. It was tagged with goat anti-mouse IgG conjugated Alexa Fluor 594 antibody (Invitrogen, USA) to exhibit red fluorescence. (a-c) Fluorescent images of monoclonal mouse anti-cyt-c antibody show that the amount of cyt-c incorporated on NPG increases with the concentration of the linker (DTSSP) utilized at (a) $2.5 \mathrm{mM}$ (b) $25 \mathrm{mM}$, and (c) $250 \mathrm{mM}$ concentrations. The control experiments $(-\mathrm{Cyt}-\mathrm{c})$ reveal that regardless of DTSSP concentration, there is no tangible attachment of the anti-cyt-c antibody to the substance. Scale bar is $50 \mu \mathrm{m}$. (d) Side by side phase contrast (left) and fluorescent (right) images of a cyt-cimmobilized NPG electrode surface reveal the peeled off film is indeed cyt-c. Scale bar is $100 \mu \mathrm{m}$. Optical snapshots of cyt-c immobilized (e) unmodified and (f) NPG-covered electrodes. Scale bar is $1 \mathrm{~mm}$. 


\section{On sensor calibration using the xanthine-XOD system}

The relatively short half-life of biologically produced superoxides would probably be the major hurdle in calibration of superoxide biosensors. ${ }^{1-6}$ Hence, a steady state source for the analyte is required. We used the xanthine-XOD system to produce continuous and computable amounts of superoxides as a function of XOD concentration and then obtained the biosensor current response versus the analyte concentration.

In a saturated xanthine solution (o.5 mM in HEPES) with ample amount of oxygen, the following enzymatic reaction produces indefinite amounts of superoxides that is quickly self-disproportionated. ${ }^{2,7}$

$$
\begin{array}{ll}
\text { Superoxide production: } & \text { xanthine }+2 \mathrm{O}_{2}+\mathrm{H}_{2} \mathrm{O} \stackrel{\text { in the presence of XOD }}{\longrightarrow} \text { urate }+2 \mathrm{H}^{+}+2 \mathrm{O}_{2}^{--} \\
\text {Superoxide disproportionation: } & 2 \mathrm{O}_{2}^{--}+2 \mathrm{H}^{+} \stackrel{k_{2}}{\rightarrow} \mathrm{O}_{2}+\mathrm{H}_{2} \mathrm{O}_{2}
\end{array}
$$

The production and disproportionation rates can be simplified to $v_{1}=2 k_{1}[\mathrm{XOD}]$, and $v_{2}=2 k_{2}\left[\mathrm{O}_{2}^{-{ }^{-}}\right]^{2}$, where [XOD] and $\left[\mathrm{O}_{2}^{-}{ }^{-}\right]$are the enzyme and superoxides concentrations in moles, and $k_{1}=2 \mathrm{~s}^{-1}$ and $k_{2}=1.5 \times 10^{5} \mathrm{M}^{-1} \mathrm{~s}^{-1}$ are the production and disproportionation rate constants, respectively. In this case, the net instantaneous accumulation rate of superoxide is:

Superoxide net generation:

$$
R(t)=\frac{d[S O A]}{d t}=v_{1}-v_{2}
$$

At steady state $R(t)=\mathrm{o}$, as a result the steady state concentration of superoxides is given by:

$$
\left[\mathrm{O}_{2}^{\bullet-}\right]=\sqrt{k_{1} / k_{2}}[\mathrm{XOD}]^{1 / 2}
$$

The enzyme data sheet reports a minimum protein content of $5 \mathrm{mg} \mathrm{mL}^{-1}$ and an activity range of 1.0 to $2.0 \mathrm{U} \mathrm{mg}^{-1}$ for bovine milk extracted XOD (X450o, Sigma, USA), which in average translates to $2.36 \mathrm{nM}$ per unit of enzyme activity. Using these data, and if [XOD] is expressed in $\mathrm{mU} \mathrm{mL}^{-1}$ instead of moles, the constant of proportionality in Eq. 4 will become $\alpha=$ $3.95 \mu \mathrm{M} \mathrm{mL}^{1 / 2} \mathrm{mU}^{-1 / 2}$. As explained in the manuscript, we used $\alpha$ to compute $\left[\mathrm{O}_{2}^{-{ }^{-}}\right]$in moles as a function of [XOD] in $\mathrm{mU}$ $\mathrm{mL}^{-1}$.

\section{On the dependence of electrode sensitivity upon surface coating}

NPG-covered electrodes without a cyt-c coating were also subjected to calibration in the xanthine/XOD system. Cyt-c-less electrodes exhibited superoxide sensitiveness of $0.389 \pm 0.008 \mathrm{nA} \mathrm{nM}^{-1} \mathrm{~cm}^{-2}$, which is less than those of similar electrodes modified with cyt-c, i.e. $S_{\text {bare NPG }}<S_{\mathrm{NPG}+\text { cyt-c }}$. The corresponding ratio of gains defined as $r=S_{\mathrm{NPG}+\text { cyt-c }} / S_{\text {bare }} \mathrm{NPG}$ was $r=4.88$ in average.

Interestingly, $r$, representing the factor over which superoxide sensitivity of a bare gold electrode scales with a DTSSP/cyt$c$ coating, match the value reported earlier on circular gold electrodes by Chen et al. ${ }^{2}$ For instance, the amperometric response of their bare gold electrode showed an unnormalized sensitivity of $S_{\mathrm{Au}}=16 \mathrm{nA} \mu \mathrm{M}^{-1}$, to superoxide (extracted from the amperogram of Figure 1 in the Supplementary Information therein). Whereas the sensitivity of their cyt-c-immobilized electrode operated in PBS was $S_{\mathrm{Au}+\text { cyt-c }}=71 \mathrm{nA} \mu \mathrm{M}^{-1}$, hence the gain ratio was $S_{\mathrm{Au}+\text { cyt-c }} / S_{\mathrm{bare} \text { Au }}=4.44$ (Identically, for our sensors the same ratio was computed to be $r_{\mathrm{NPG}}=4.88$ ).

Moreover, these results show that irrespective of the presence of cyt-c, superoxides are predominantly adsorbed and reacted at the surface of the NPG film rather than diffusing deep into the bulk. Otherwise the value of $r$ would have been different from those reported on circular gold electrodes by Chen et al.

\section{On evaluating the low limits of detection}

Low limits of detection (LOD) where computed for the biosensors of both type using

$$
\mathrm{LOD}=3 \sigma_{\text {low }} / S_{\text {ave }}
$$

where $\sigma_{\text {low }}$ and $S_{\text {ave }}$ are the standard deviation of amperometric sensor readouts at the lowest detectable $\left[\mathrm{O}_{2}^{-{ }^{-}}\right]$and the average biosensor sensitivity respectively. ${ }^{8}$ 

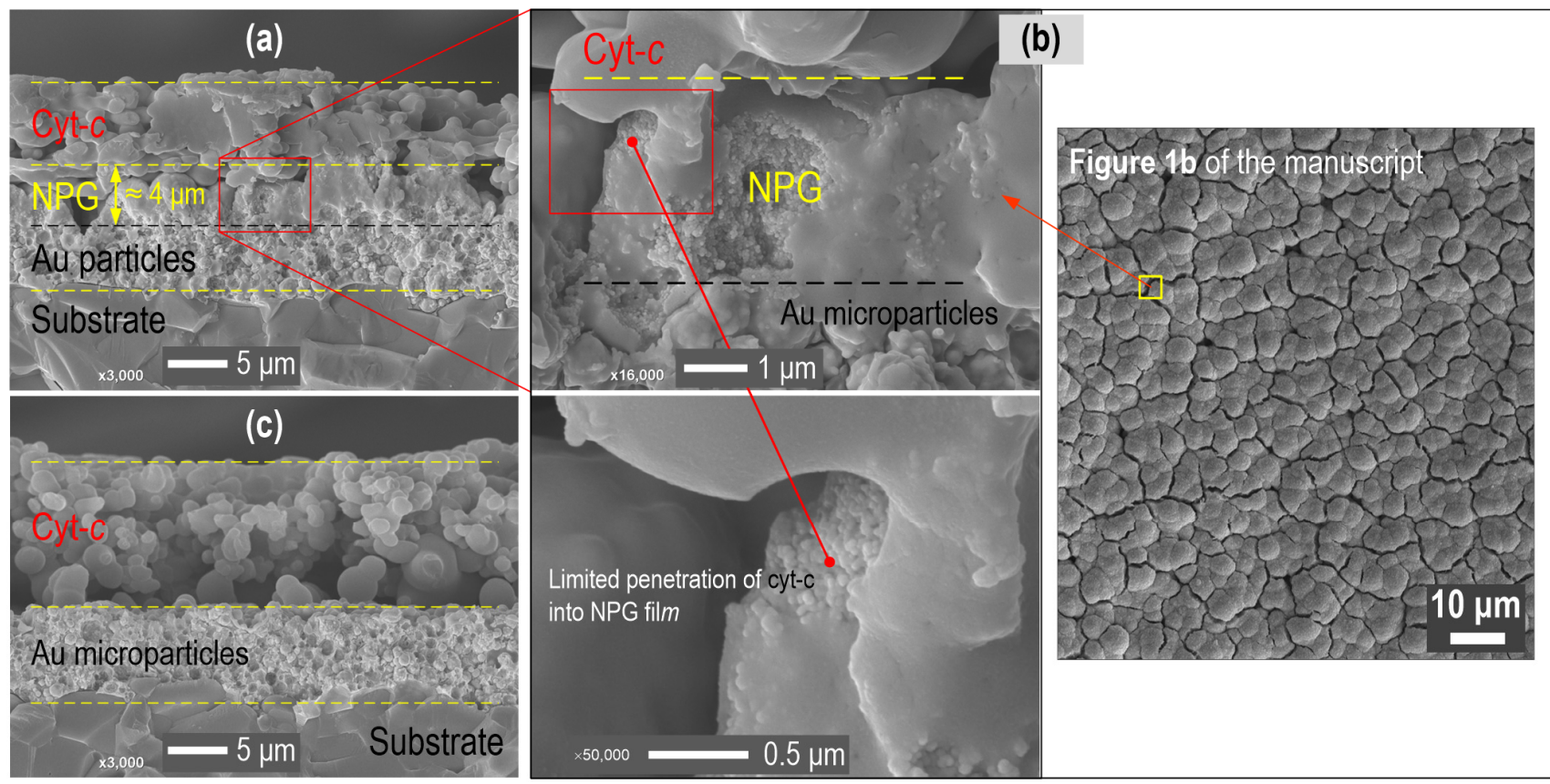

Figure S5. Cross-sectional SEM images snapped from the electrodes of both kind after cyt-c immobilization. (a) Close-up of an NPG-covered electrode and (b) magnified views of the gold particles/NPG/cyt-c interface, showing the coverage of the protein over the NPG film. Cyt-c has diffused through the cracks on the NPG film, as shown in Figure $1 \mathrm{~b}$ of the manuscript, and covered the pores subsequently. (c) Close-up of an unmodified electrode.
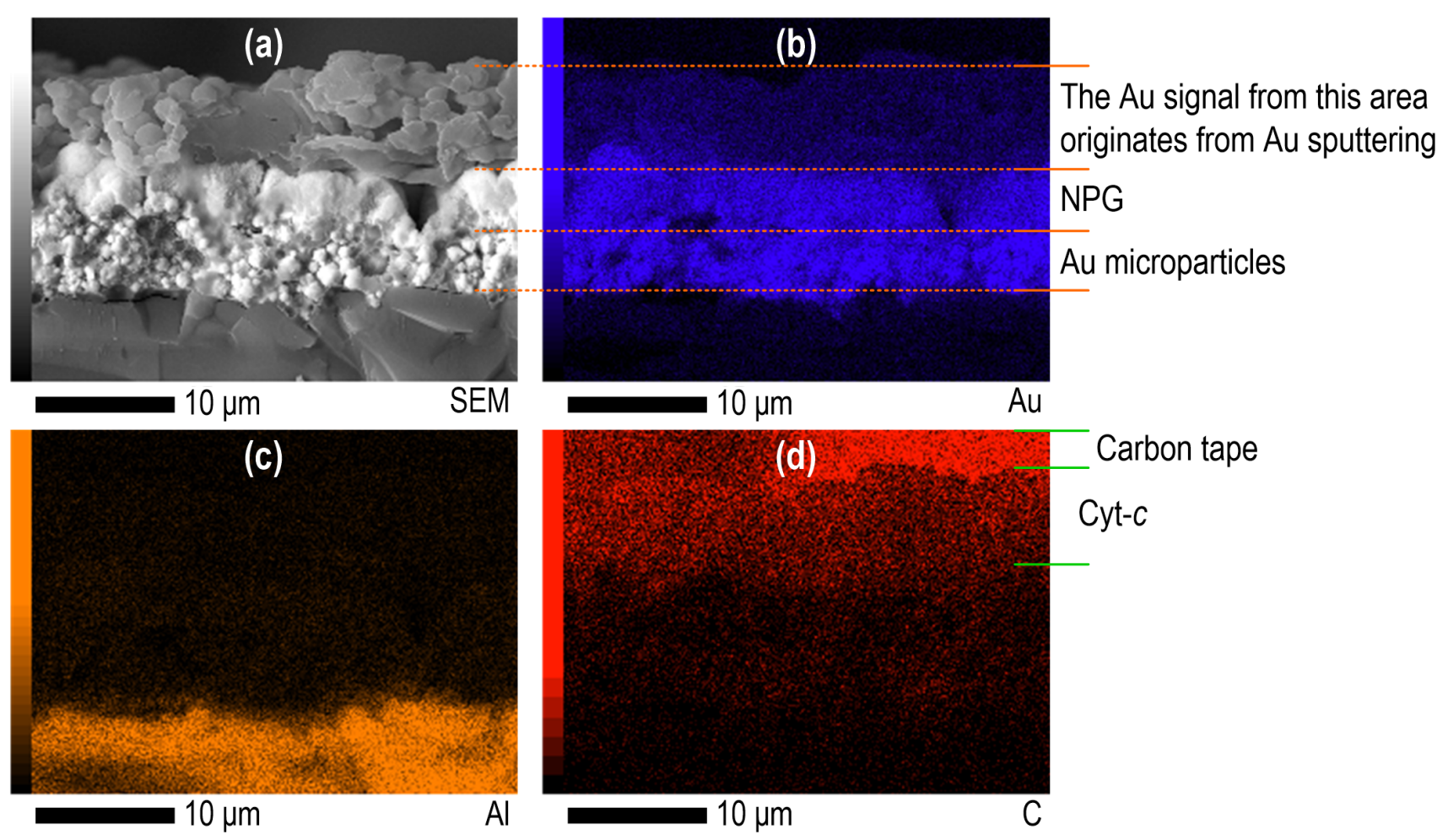

Figure S6. EDS map of the NPG-covered electrode cross-section with cyt-c. (a) SEM image as a reference, (b) gold signal (notice that the background gold signal emitted from the cyt-c area is originated from the sputtered gold layer deposited to make the sample conductive for SEM observations), (c) Al signal from the alumina substrate, and (d) carbon from the protein and the carbon tape used to fix the sample. Apparently, cyt-c has partially diffused into the NPG film. 

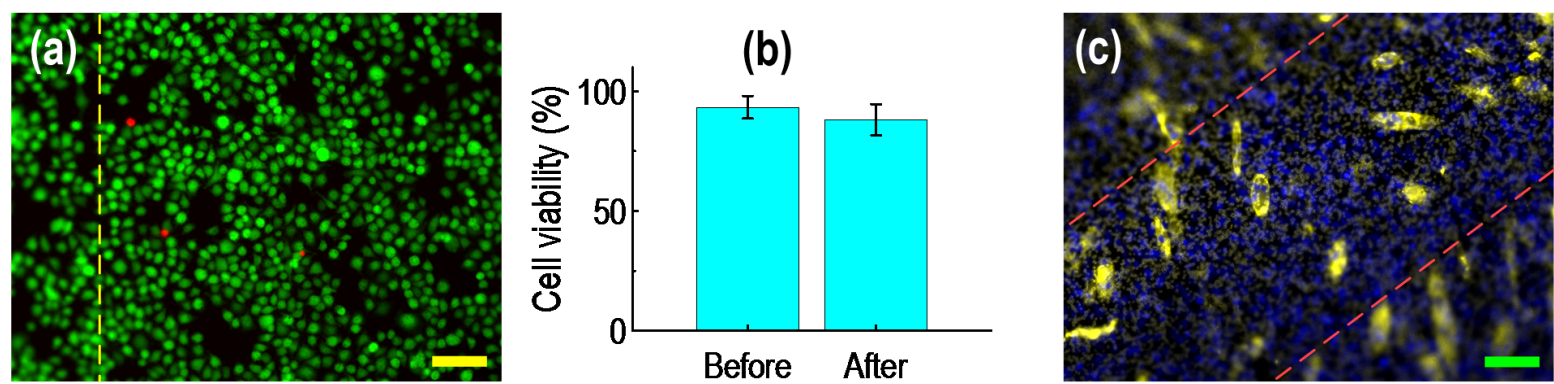

Figure $\mathrm{S}_{7} \cdot \mathrm{C}_{2} \mathrm{C}_{12}$ cells seeded on polyester porous membranes. (a) Fluorescent image of $\mathrm{C}_{2} \mathrm{C}_{12}$ myoblasts cultured on porous polyester membranes and stained using live/dead assay (Invitrogen, USA) comprised of calcein-AM for live and ethidium homodimer-1 for dead cells. These cells were dyed immediately after performing the electrochemical measurements. ImageJ utility (v.1.46r, $\mathrm{NIH}$ ) was used to count the total number of live (green) and dead (red) cells and compute the live/dead ratio as a measure of viability. (b) Cell viabilities in percent obtained before and after the tests elucidate the nontoxic nature of the electrochemical measurement process. (c) Fluorescent image of immunostained $\mathrm{C}_{2} \mathrm{C}_{12}$ myotubes captured to reveal cell nuclei (blue) and myosin heavy chain (yellow). As indicated by dashed lines, a wide-area focus was impossible to achieve due to membrane wrinkling. Myotubes at day 11 of differentiation were identified using anti-fast skeletal myosin antibody (MY32) (Abcam, USA) latched to Alexa Fluor 488-conjugated anti-IgG antibody (Invitrogen, USA) and the nuclei were revealed using 4,6-diamidino-2-phenylindole (DAPI) (Sigma, USA) according to the following procedure. The cells were first fixed in $3.7 \%$ formaldehyde solution (Sigma, USA), permeabilized with $0.3 \%$ Triton X-10o (Sigma, USA), soaked in 5\% BSA solution for 15 min to inhibit unspecific antibody binding, and then treated with $\mathrm{MY}_{32}$ diluted 1000 times in o.1\% BSA and left overnight at $4{ }^{\circ} \mathrm{C}$. After washing the samples thoroughly two times with PBS, Alexa Fluor 488-conjugated anti-IgG antibody and DAPI both diluted 1000 times, were added to the medium and then the samples were incubated for one hour in $37^{\circ} \mathrm{C}$ before microscopy. All solutions were PBS-based. Scale bars are $100 \mu \mathrm{m}$ in (a) and (c). Cell-carrying membranes were treated by Hoechst 33342 (ThermoFisher Scientific, USA) $(1 \mu \mathrm{M})$ and incubated at $37^{\circ} \mathrm{C}$ for 30 min to reveal the cell nuclei for quantification purposes (not shown). Stained cells were counted using ImageJ.

(a)

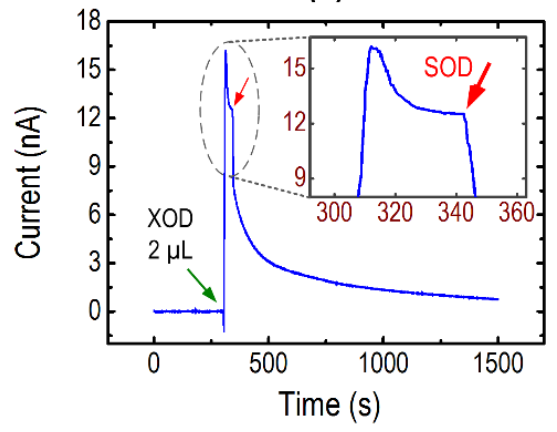

(b)

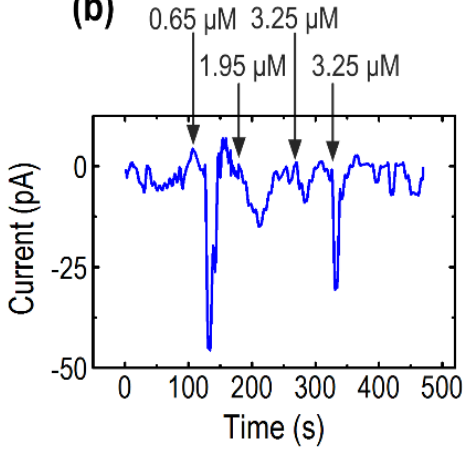

Figure S8. Biosensor specificity demonstrated by the superoxide-scavenging effect of SOD. (a) SOD dismutates superoxides produced by xanthine/XOD reaction. As shown by the arrow, addition of $1000 \mathrm{U} \mathrm{mL}^{-1}$ of SOD after XOD was added to a saturated solution of xanthine, caused an abrupt drop in the current. Since the enzymatic reaction is strong, superoixde production is resumed after a while. The amperogram was recorded using an NPG-covered electrode sensor operating at $350 \mathrm{mV}$. (b) When SOD was added to the medium at $200 \mathrm{U} \mathrm{mL}^{-1}$, no superoxide signal was recorded from myoblasts at day 2 of culture (at $200 \mathrm{mV}$ ). Arrows show the moments/dosages at which PMA was added. 


\section{References}

(1) Calas-Blanchard, C.; Catanante, G.; Noguer, T. Electrochemical Sensor and Biosensor Strategies for ROS/RNS Detection in Biological Systems. Electroanalysis 2014, 26, 1277-1286.

(2) Chen, X. J.; West, A. C.; Cropek, D. M.; Banta, S. Detection of the Superoxide Radical Anion Using Various Alkanethiol Monolayers and Immobilized Cytochrome c. Anal. Chem. 2008, 8o, 9622-9629.

(3) Gomes, A.; Fernandes, E.; Lima, J. L. F. C. Fluorescence probes used for detection of reactive oxygen species. J. Biochem. Biophys. Methods 2005, 65, 45-80.

(4) Manjare, S. T.; Kim, S.; Heo, W. D.; Churchill, D. G. Selective and Sensitive Superoxide Detection with a New Diselenide-Based Molecular Probe in Living Breast Cancer Cells. Org. Lett. 2014, 16, 410-412.

(5) Fabian, R. H.; DeWitt, D. S.; Kent, T. A. In Vivo Detection of Superoxide Anion Production by the Brain Using a Cytochrome Electrode. J. Cereb. Blood Flow Metab. 1995, 15, 242-247.

(6) Ganesana, M.; Erlichman, J. S.; Andreescu, S. Real-time monitoring of superoxide accumulation and antioxidant activity in a brain slice model using an electrochemical cytochrome c biosensor. Free Radical Biol. Med. 2012, 53, 2240-2249.

(7) Tammeveski, K.; Tenno, T. T.; Mashirin, A. A.; Hillhouse, E. W.; Manning, P.; McNeil, C. J. Superoxide electrode based on covalently immobilized cytochrome c: modelling studies. Free Radical Biol. Med. 1998, 25, 973-978.

(8) Validation of Analytical Procedures: Text and Methodology Q2(R1). FDA, Federal Register: 1997; vol. 62, pp $27463-27467$. 